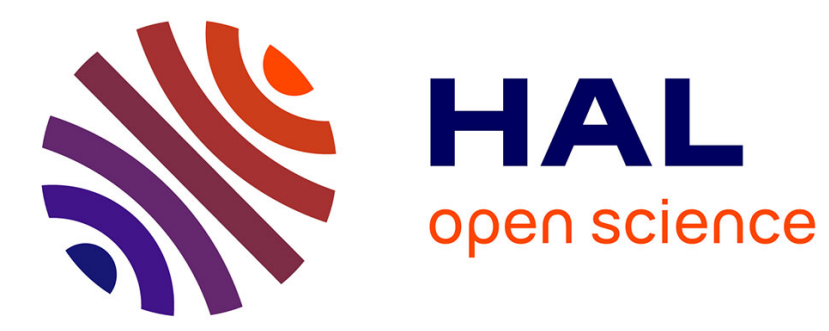

\title{
Optimal convergence analysis for the eXtended Finite Element Method
}

Serge Nicaise, Yves Renard, Elie Chahine

\section{To cite this version:}

Serge Nicaise, Yves Renard, Elie Chahine. Optimal convergence analysis for the eXtended Finite Element Method. 2008. hal-00339853v1

\section{HAL Id: hal-00339853 \\ https://hal.science/hal-00339853v1}

Preprint submitted on 19 Nov 2008 (v1), last revised 6 Jun 2018 (v2)

HAL is a multi-disciplinary open access archive for the deposit and dissemination of scientific research documents, whether they are published or not. The documents may come from teaching and research institutions in France or abroad, or from public or private research centers.
L'archive ouverte pluridisciplinaire HAL, est destinée au dépôt et à la diffusion de documents scientifiques de niveau recherche, publiés ou non, émanant des établissements d'enseignement et de recherche français ou étrangers, des laboratoires publics ou privés. 


\title{
Optimal convergence analysis for the eXtended Finite Element Method
}

\author{
Serge Nicaise ${ }^{1}$, Yves Renard ${ }^{2}$, Elie Chahine ${ }^{3}$
}

\begin{abstract}
We establish some optimal a priori error estimate on some variants of the eXtended Finite Element Method (Xfem), namely the Xfem with a cut-off function and the standard Xfem with a fixed enrichment area. The results are established for the Lamé system (homogeneous isotropic elasticity) and the Laplace problem. The convergence of the numerical stress intensity factors is also investigated. We show some numerical experiments which corroborate the theoretical results.
\end{abstract}

Keywords: extended finite element method, error estimates, stress intensity factors.

\section{Introduction}

Inspired by the Pufem [26], the Xfem (extended finite element method) was introduced by Moës et al. in 1999 [28, 27] for plane linear isotropic elasticity problems (Lamé system) in cracked domains. The main advantage of this method is the ability to take into account the discontinuity across the crack and the asymptotic displacement at the crack tip by addition of special functions into the finite element space. It allows the use of a mesh which is independent of the geometry of the crack. This avoids the remeshing operations when the crack propagates and the corresponding re-interpolation operations which can cause numerical instabilities. In the original method, the asymptotic displacement is incorporated into the finite element space multiplied by the shape function of a background Lagrange finite element method. However, we deal also with a variant, introduced in [12], where the asymptotic displacement is multiplied by a cut-off function. This variant is similar to the classical singular enrichment method introduced in 1973 by Strang and Fix [32] but it additionally preserves the independence of the mesh to the geometry of the crack which is indeed the essential contribution of Xfem.

Another classical method to take into account a singular behavior of the solution is the dual singular function method introduced by M. Dobrowolski et al. in [5] (see also [19, 10]) or a more recent variant the singular complement method introduced by P. Ciarlet Jr. et al. in [17] (for a $L$-shape domain, see [29]). These methods require the use of dual singular functions which can be difficult to obtain in some situations (even for the Lamé system) or quite impossible to obtain when just the asymptotic behavior is known (for non-linear elasticity [2] or Mindlin plate model for instance).

The Xfem strategy can be adapted to various situations. See among many other references $[3,6,7,8,23,25,36,37,35,38]$. In particular, a fictitious domain method can be

\footnotetext{
${ }^{1}$ Université de Valenciennes et du Hainaut Cambrésis, LAMAV, FR CNRS 2956, Institut des Sciences et Techniques de Valenciennes, F-59313 - Valenciennes Cedex 9 France, email: Serge.Nicaise@univvalenciennes.fr

${ }^{2}$ Université de Lyon, CNRS

INSA-Lyon, ICJ UMR5208, LaMCoS UMR5259, F-69621, Villeurbanne, France, Yves.Renard@insa-lyon.fr

${ }^{3}$ Laboratory for Nuclear Materials, Nuclear Energy and Safety Research Department, Paul Scherrer Institute OVGA/14, CH-5232 Villigen PSI, Switzerland, elie.chahine@psi.ch.
} 
derived from the principle of Xfem (see [24, 4]) and it is possible to adapt some strategies when the asymptotic behavior is unknnown or only partially known (see $[11,13,14]$ ).

In the present paper, we improve the results given in [12] concerning the variant which uses a cut-off function. We also give some additional error estimates concerning the stress intensity factors and the standard Xfem. The theoretical results are established for both the Lamé system and the Laplace problem. Some numerical tests that illustrate and confirm the theoretical results are presented.

\section{The model problems}

The analysis will be performed on a cracked domain $\Omega \subset \mathbb{R}^{2}$ for two model problems: The Laplace equation and the Lamé system. The crack $\Gamma_{C}$ is assumed to be straight. In both cases, the boundary $\partial \Omega$ of $\Omega$ is partitioned into $\Gamma_{D}, \Gamma_{N}$ and $\Gamma_{C}$ (see Fig. 1). A Dirichlet condition is prescribed on $\Gamma_{D}$, a Neumann one on $\Gamma_{N}$ while on the crack $\Gamma_{C}$ we consider an homogeneous Neumann condition.

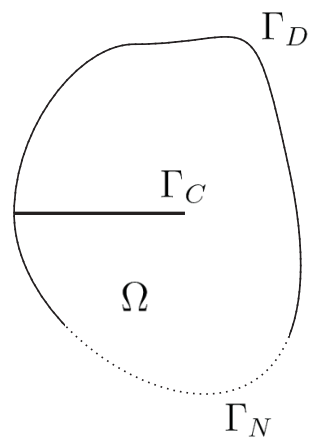

Figure 1: The cracked domain $\Omega$.

The weak formulation of the (scalar) Laplace equation on this domain reads as follows:

$$
\left\{\begin{array}{l}
\text { Find } u \in V \quad \text { such that } a(u, v)=l(v) \quad \forall v \in V, \\
a(u, v)=\int_{\Omega} \nabla u \cdot \nabla v d x \\
l(v)=\int_{\Omega} f v d x+\int_{\Gamma_{N}} g v d \Gamma \\
V=\left\{v \in H^{1}(\Omega) ; v=0 \text { on } \Gamma_{D}\right\} .
\end{array}\right.
$$

While the one of the Lamé (vectorial) system (linear elasticity problem on this domain for an isotropic material) is:

$$
\left\{\begin{array}{l}
\text { Find } \quad u \in V \quad \text { such that } a(u, v)=l(v) \quad \forall v \in V, \\
a(u, v)=\int_{\Omega} \sigma(u): \varepsilon(v) d x \\
l(v)=\int_{\Omega} f \cdot v d x+\int_{\Gamma_{N}} g \cdot v d \Gamma \\
\sigma(u)=\lambda \operatorname{tr}(\varepsilon(u)) I+2 \mu \varepsilon(u), \\
V=\left\{v \in H^{1}\left(\Omega ; \mathbb{R}^{2}\right) ; v=0 \text { on } \Gamma_{D}\right\}
\end{array}\right.
$$

where $\sigma(u)$ denotes the stress tensor, $\varepsilon(u)=\frac{1}{2}\left(\nabla u+\nabla u^{T}\right)$ is the linearized strain tensor, 
$f$ and $g$ are some external load densities on $\Omega$ and $\Gamma_{N}$ respectively, and $\lambda>0, \mu>0$ are the Lamé coefficients.

In both cases, we suppose $\bar{\Omega}, f$ and $g$ smooth enough for the solution $u$ of Problem (1) or (2) to be written as a sum of a singular part $u_{s}$ and a regular part $u-u_{s}$ (see $[22,21]$ ) satisfying:

$$
u-u_{s} \in H^{2}\left(\Omega ; \mathbb{R}^{d}\right),
$$

with

$$
d=1 \quad \text { and } \quad u_{s}=K_{L} u_{L}
$$

for the solution to the Laplace equation (1), and

$$
d=2 \quad \text { and } \quad u_{s}=K_{I} u_{I}+K_{I I} u_{I I},
$$

for the solution to the Lamé system (2). The scalars $K_{L}, K_{I}$ and $K_{I I}$ are the so-called stress intensity factors and the functions $u_{L}, u_{I}$ and $u_{I I}$ are given in polar coordinates relatively to the crack tip (Fig. 2) by:

$$
\begin{aligned}
u_{L}(r, \theta) & =\sqrt{r} \sin \frac{\theta}{2}, \\
u_{I}(r, \theta) & =\frac{1}{E} \sqrt{\frac{r}{2 \pi}}(1+\nu)\left(\begin{array}{c}
\cos \frac{\theta}{2}(\delta-\cos \theta) \\
\sin \frac{\theta}{2}(\delta-\cos \theta)
\end{array}\right), \\
u_{I I}(r, \theta) & =\frac{1}{E} \sqrt{\frac{r}{2 \pi}}(1+\nu)\left(\begin{array}{c}
\sin \frac{\theta}{2}(\delta+2+\cos \theta) \\
\cos \frac{\theta}{2}(\delta-2+\cos \theta)
\end{array}\right),
\end{aligned}
$$

where $\nu=\frac{\lambda}{\lambda+2 \mu}$ denotes the Poisson ratio, $E=\frac{4 \mu(\lambda+\mu)}{\lambda+2 \mu}$ the Young modulus and $\delta=3-4 \nu$ (plane stress approximation). Note that $u_{L}, u_{I}$ and $u_{I I}$ belong to $H^{3 / 2-\eta}(\Omega)$ for any $\eta>0$ (see [22]) which limits the order of the convergence rate of a classical finite element method to $O\left(h^{1 / 2}\right)$ where $h$ is the mesh parameter.

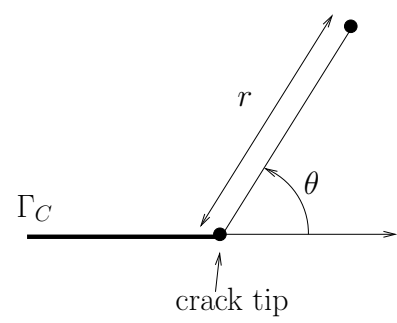

Figure 2: Polar coordinates respectively to the crack tip $\Omega$.

\section{Xfem with a cut-off function}

The Xfem variant which uses a cut-off function was proposed in [12]. The principle of the standard Xfem (see $[28,27]$ ) is to consider a mesh independent of the crack geometry. An Heaveaside type function is used to represent the discontinuity across the straight crack:

$$
H(x)= \begin{cases}+1 & \text { if }\left(x-x^{*}\right) \cdot n \geq 0 \\ -1 & \text { elsewhere }\end{cases}
$$


where $x^{*}$ denotes the crack tip and $n$ is a given normal to the crack. Moreover, the nonsmooth functions $u_{L}, u_{I}$ and $u_{I I}$ are integrated to the discrete space to take into account the asymptotic behavior at the crack tip.

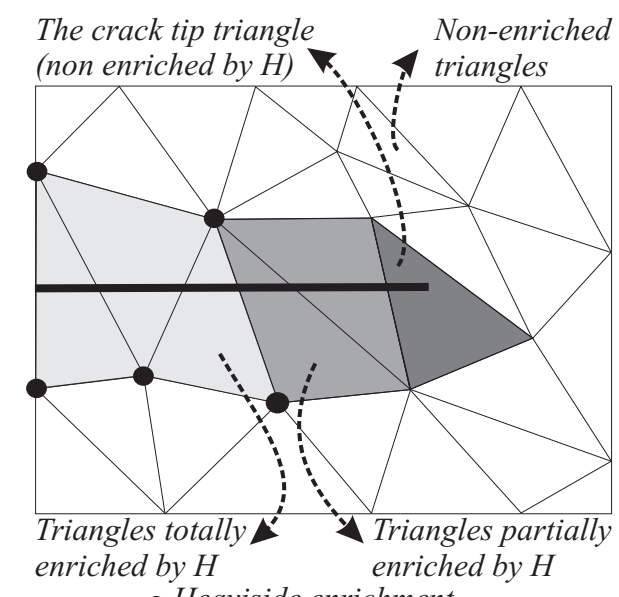

Figure 3: Enrichment strategy.

We consider an affine Lagrange finite element method defined on a regular triangulation $\mathscr{T}_{h}$ (in the sense of the Ciarlet [16]) of the non-cracked domain $\bar{\Omega}, h$ being the mesh parameter i.e. the largest diameter of the elements of $\mathscr{T}_{h}$. The piecewise $P_{1}$ basis functions are denoted $\varphi_{i}$. In this section, We consider the variant of Xfem proposed in [12] for which a whole area around the crack tip is enriched by using a cut-off function denoted $\chi$. The approximation of the Laplace equation reads as

$$
\left\{\begin{array}{l}
\text { Find } \quad u^{h} \in V^{h} \quad \text { such that } a\left(u^{h}, v^{h}\right)=l\left(v^{h}\right) \quad \forall v^{h} \in V^{h}, \\
a\left(u^{h}, v^{h}\right)=\int_{\Omega} \nabla u^{h} \cdot \nabla v^{h} d x, \\
l\left(v^{h}\right)=\int_{\Omega} f v^{h} d x+\int_{\Gamma_{N}} g v^{h} d \Gamma, \\
V^{h}=\left\{v^{h}=\sum_{i \in I} a_{i} \varphi_{i}+\sum_{i \in I_{H}} b_{i} H \varphi_{i}+K_{L, h} \chi u_{L} ; a_{i}, b_{i}, K_{L, h} \in \mathbb{R}\right\} .
\end{array}\right.
$$

where $I$ is the set of node indices of the $P_{1}$ finite element method, $I_{H}$ is the sub-set of node indices whose corresponding shape functions have their supports completely cut by the crack and $\chi$ is a $W^{2, \infty}(\bar{\Omega})$ cut-off function verifying for fixed $0<r_{0}<r_{1}$

$$
\left\{\begin{array}{l}
\chi(r)=1 \text { if } r<r_{0} \\
0<\chi(r)<1 \text { if } r_{0}<r<r_{1}, \\
\chi(r)=0 \text { if } r_{1}<r
\end{array}\right.
$$

Concerning now the Lamé system, we consider two different ways to incorporate the asymptotic displacement. The first one is directly based on a vectorial enrichment with $u_{I}$ and 


$$
\begin{aligned}
& u_{I I} \text { : } \\
& \left\{\begin{array}{l}
\text { Find } \quad u^{h} \in V^{h} \quad \text { such that } a\left(u^{h}, v^{h}\right)=l\left(v^{h}\right) \quad \forall v^{h} \in V^{h}, \\
a\left(u^{h}, v^{h}\right)=\int_{\Omega} \sigma\left(u^{h}\right): \varepsilon\left(v^{h}\right) d x, \\
l\left(v^{h}\right)=\int_{\Omega} f \cdot v^{h} d x+\int_{\Gamma_{N}} g \cdot v^{h} d \Gamma, \\
\sigma\left(u^{h}\right)=\lambda \operatorname{tr} \varepsilon\left(u^{h}\right) I+2 \mu \varepsilon\left(u^{h}\right), \\
V^{h}=\left\{v^{h}=\sum_{i \in I} a_{i} \varphi_{i}+\sum_{i \in I_{H}} b_{i} H \varphi_{i}+K_{I, h} \chi u_{I}+K_{I I, h} \chi u_{I I} ; a_{i}, b_{i} \in \mathbb{R}^{2}, K_{I, h}, K_{I I, h} \in \mathbb{R}\right\} .
\end{array}\right.
\end{aligned}
$$

The second one corresponds to a more classical Xfem approximation with a scalar enrichement of each component:

$$
\left\{\begin{array}{l}
\text { Find } u^{h} \in V^{h} \quad \text { such that } a\left(u^{h}, v^{h}\right)=l\left(v^{h}\right) \quad \forall v^{h} \in V^{h}, \\
a\left(u^{h}, v^{h}\right)=\int_{\Omega} \sigma\left(u^{h}\right): \varepsilon\left(v^{h}\right) d x, \\
l\left(v^{h}\right)=\int_{\Omega} f \cdot v^{h} d x+\int_{\Gamma_{N}} g \cdot v^{h} d \Gamma, \\
\sigma\left(u^{h}\right)=\lambda \operatorname{tr} \varepsilon\left(u^{h}\right) I+2 \mu \varepsilon\left(u^{h}\right), \\
V^{h}=\left\{v^{h}=\sum_{i \in I} a_{i} \varphi_{i}+\sum_{i \in I_{H}} b_{i} H \varphi_{i}+\sum_{j=1}^{4} c_{j} F_{j} \chi ; a_{i}, b_{i}, c_{j} \in \mathbb{R}^{2}\right\},
\end{array}\right.
$$

where the set of functions $\left\{F_{j}(x)\right\}_{1 \leq j \leq 4}$ is defined by

$$
\left\{F_{j}(x)\right\}_{1 \leq j \leq 4}=\left\{\sqrt{r} \sin \frac{\theta}{2}, \sqrt{r} \cos \frac{\theta}{2}, \sqrt{r} \sin \frac{\theta}{2} \cos \theta, \sqrt{r} \cos \frac{\theta}{2} \cos \theta\right\} .
$$

Note that the nonsmooth functions $u_{I}$ and $u_{I I}$ can be decomposed on this set of functions.

\section{Optimal Error estimate for the Xfem with a cut-off func- tion}

We use the notation $a \lesssim b$ to signify that there exists a constant $C>0$ independent of the mesh parameter and of the solution such that $a \leq C b$. For a non negative real number $s$ let $H^{s}(D)$ denote the standard Sobolev space of order $s$ in $D$ of norm (resp. semi-norm) denoted by $\|\cdot\|_{s, D}\left(\right.$ resp. $\left.|\cdot|_{s, D}\right)$, see for instance [1].

The aim of this section is to establish the following result which is the optimal version of Theorem 1 in [12]:

Theorem 1 Assume that the displacement field u, solution to Problem (1) (resp. Problem (2)), satisfies Condition (3). Then, the following estimate holds

$$
\left\|u-u^{h}\right\|_{1, \Omega} \lesssim h\left\|u-\chi u_{s}\right\|_{2, \Omega},
$$

where $u^{h}$ is the solution to Problem (10) (resp. to Problem (12) or to Problem (13)), $u_{s}$ is the singular part of $u\left(\right.$ see (3)) and $\chi$ is the $W^{2, \infty}(\Omega)$ cut-off function introduced before.

The outline of the proof globally follows the one of Theorem 1 in [12]. Some sub-optimal intermediary results are here replaced by optimal ones. 
We recall the definition of the adapted interpolation operator $\Pi^{h}$. The interpolation error estimates are then computed locally over every different type of triangles: triangles totally enriched by the Heaveaside function, triangles partially enriched by the Heaviside function and the triangle containing the crack-tip.

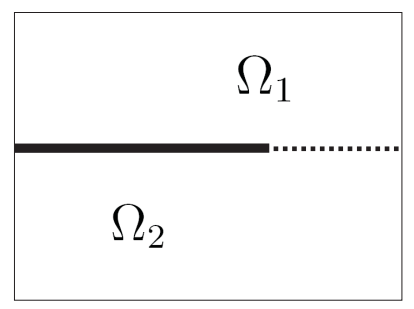

Figure 4: Domain decomposition.

The domain $\Omega$ is divided into $\Omega_{1}$ and $\Omega_{2}$ according to the crack and a straight extension of the crack (Fig.4) such that the value of $H$ is $(-1)^{k}$ on $\Omega_{k}, k=1,2$. Let us denote $u_{r}=u-\chi u_{s}$, and $u_{r}^{k}$ the restriction of $u_{r}$ to $\Omega_{k}, k \in\{1,2\}$. Then, there exists in $H^{2}\left(\bar{\Omega} ; \mathbb{R}^{d}\right)$ an extension $\widetilde{u}_{r}^{k}$ of $u_{r}^{k}$ on $\bar{\Omega}$ such that (see [1])

$$
\begin{aligned}
\left\|\widetilde{u}_{r}^{1}\right\|_{2, \bar{\Omega}} & \lesssim\left\|u_{r}^{1}\right\|_{2, \Omega_{1}}, \\
\left\|\widetilde{u}_{r}^{2}\right\|_{2, \bar{\Omega}} & \lesssim\left\|u_{r}^{2}\right\|_{2, \Omega_{2}} .
\end{aligned}
$$

Definition 1 (from [12]) Given a displacement field u satisfying (3) and two extensions $\widetilde{u}_{r}^{1}$ and $\widetilde{u}_{r}^{2}$ respectively of $u^{1}$ and $u^{2}$ in $H^{2}\left(\Omega ; \mathbb{R}^{d}\right)$, we define $\Pi^{h} u$ as the element of $V^{h}$ such that

$$
\Pi^{h} u=\sum_{i \in I} a_{i} \varphi_{i}+\sum_{i \in I_{H}} b_{i} H \varphi_{i}+\chi u_{s}
$$

where $a_{i}, b_{i}$ are given as follows ( $x_{i}$ denotes the node associated to $\left.\varphi_{i}\right)$ :

$$
\begin{aligned}
& \text { if } i \in\left\{I \backslash I_{H}\right\} \text { then } a_{i}=u_{r}\left(x_{i}\right), \\
& \text { if } i \in I_{H} \text { and } x_{i} \in \bar{\Omega}_{k} \text { then }(k \in\{1,2\}, l \neq k)\left\{\begin{array}{l}
a_{i}=\frac{1}{2}\left(u_{r}^{k}\left(x_{i}\right)+\widetilde{u}_{r}^{l}\left(x_{i}\right)\right), \\
b_{i}=\frac{1}{2}\left(u_{r}^{k}\left(x_{i}\right)-\widetilde{u}_{r}^{l}\left(x_{i}\right)\right)(-1)^{k} .
\end{array}\right.
\end{aligned}
$$

From this definition, the following result holds:

Lemma 1 (from [12]) The function $\Pi^{h} u$ satisfies

(i) $\Pi^{h} u=I^{h} u_{r}+\chi u_{s}$ over a triangle non-enriched by $H$,

(ii) $\left.\Pi^{h} u\right|_{K \cap \Omega_{k}}=I^{h} \widetilde{u}_{r}^{k}+\chi u_{s}$ over a triangle $K$ totally enriched by $H$,

where $I^{h}$ denotes the classical interpolation operator for the associated finite element method.

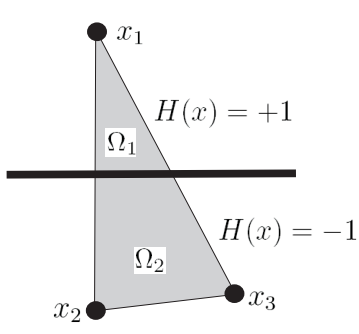

(a)

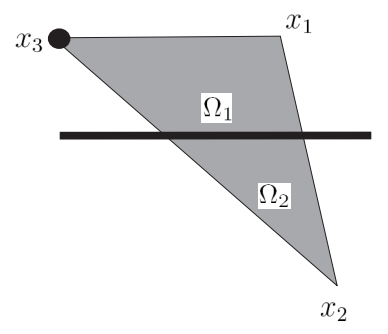

(b)

Figure 5: (a) Totally enriched triangle and (b) partially enriched triangle (Fig.3). 
For $K$ a subset of $\Omega$, we denote $h_{K}=\operatorname{diam}(K)=\max _{x_{1}, x_{2} \in K}\left|x_{1}-x_{2}\right|$ and $\rho_{K}=$ $\left\{\sup (\operatorname{diam}(B)) ; B\right.$ ball of $\left.\mathbb{R}^{2}, B \subset K\right\}$. The following lemma, established in [12], derives simply from the classical interpolation of the extensions of $u_{r}^{1}$ and $u_{r}^{2}$.

Lemma 2 (from [12]) Let $\mathscr{T}_{h}^{H}$ be the set of triangles totally enriched by $H$ (Fig.3) and $\sigma_{K}=h_{K} \rho_{K}^{-1}$. For all $K$ in $\mathscr{T}_{h}^{H}$, and for all u satisfying (3) we have the estimates

$$
\left\|u-\Pi^{h} u\right\|_{1, K \cap \Omega_{1}} \lesssim h_{K} \sigma_{K}\left\|\widetilde{u}_{r}^{1}\right\|_{2, K}
$$

and

$$
\left\|u-\Pi^{h} u\right\|_{1, K \cap \Omega_{2}} \lesssim h_{K} \sigma_{K}\left\|\widetilde{u}_{r}^{2}\right\|_{2, K}
$$

The optimal convergence is of course obtained for non enriched triangles. It remains to treat the partially enriched triangles and the triangle containing the crack tip. We will now detailled the optimal intermediary results which are original in this paper.

Let us start with the Laplace equation and recall that in that case $u_{r}^{1}$ and $u_{r}^{2}$ satisfy

$$
\partial_{n} u_{r}^{1}=\partial_{n} u_{r}^{2}=0 \text { on } \Gamma_{C} .
$$

Since the extension $\tilde{u}_{r}^{1}$ and $\tilde{u}_{r}^{2}$ are $H^{2}(\bar{\Omega})$ extension, they also satisfy the Neumann boundary condition on $\Gamma_{C}$, namely

$$
\partial_{n} \tilde{u}_{r}^{1}=\partial_{n} \tilde{u}_{r}^{2}=0 \text { on } \Gamma_{C} .
$$

We now give the main technical result.

Lemma 3 Assume that $x_{1} \in \Omega_{1}$ is a node belonging to a triangle $K$ containing the crack tip. Then

$$
\left|u_{r}^{1}\left(x_{1}\right)-\tilde{u}_{r}^{2}\left(x_{1}\right)\right| \lesssim h_{K}\left|\tilde{u}_{r}^{1}-\tilde{u}_{r}^{2}\right|_{2, B\left(0, h_{K}\right)}
$$

Proof: For shortness write $v=\tilde{u}_{r}^{1}-\tilde{u}_{r}^{2}$. Using a Taylor expansion, we have

$$
v\left(x_{1}\right)=\int_{0}^{1}\left(x_{1}^{(1)} \partial_{1} v\left(t x_{1}\right)+x_{1}^{(2)} \partial_{2} v\left(t x_{1}\right)\right) d t
$$

where $x_{1}=\left(x_{1}^{(1)}, x_{1}^{(2)}\right)$. Without loss of generality and modulo an orthonormal change of coordinates we assume that the position of the crack tip is $(0,0)$ and the crack $\Gamma_{C}$ is a part of $\left(\mathbb{R}_{-}, 0\right)$. By setting $v^{(1)}=\partial_{1} v$ and $v^{(2)}=\partial_{2} v$, and making the change of variable $s=t x_{1}$, the above identity is equivalent to

$$
v\left(x_{1}\right)=\int_{e}\left(n_{2} v^{(1)}(s)-n_{1} v^{(2)}(s)\right) d s,
$$

where $e$ is the edge joining the crack tip and $x_{1}$ and $n=\left(n_{1}, n_{2}\right)$ is (one of) the normal vector to $e$. Denote by $C$ the truncated sector determined by $e$ and the crack:

$$
C=\left\{(r \cos \theta, r \sin \theta): 0<r<h_{1} \quad \theta_{0}<\theta<\pi\right\},
$$

when $x_{1}=\left(h_{1} \cos \theta_{0}, h_{1} \sin \theta_{0}\right)$, see Fig. 6. Now setting $e_{2}=\left\{\left(h_{1} \cos \theta, h_{1} \sin \theta\right): \theta_{0}<\theta<\right.$ $\pi\}$, by Green's formula we remark that

$$
\int_{C} \partial_{1} v^{(2)} d x=\int_{\partial C} n_{1} v^{(2)} d s=\int_{e} n_{1} v^{(2)} d s+\int_{e_{2}} n_{1} v^{(2)} d s,
$$

because $n_{1}=0$ on $\Gamma_{C}$. Hence

$$
\int_{e} n_{1} v^{(2)} d s=\int_{C} \partial_{1} v^{(2)} d x-\int_{e_{2}} n_{1} v^{(2)} d s .
$$


The first term of this right-hand side will be estimated by a simple Cauchy-Schwarz inequality. For the second term by a scaling argument, we show that

$$
\int_{e_{2}}\left|v^{(2)}\right| d s \lesssim h_{1}\left\|\nabla v^{(2)}\right\|_{B\left(0, h_{1}\right)}
$$

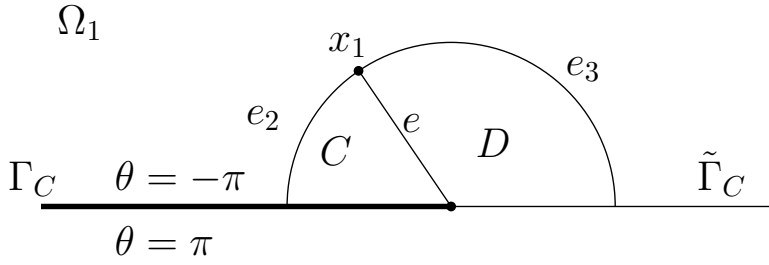

$\Omega_{2}$

Figure 6: The truncated sector $C$.

Indeed by construction $v^{(2)}$ satisfies

$$
v^{(2)}=0 \text { on } \Gamma_{C} \text {. }
$$

Therefore the change of variable $x=h_{1} \hat{x}$ maps $B\left(0, h_{1}\right)$ to the unit ball. By setting $\hat{v}^{(2)}(\hat{x})=v^{(2)}(x)$, we deduce that

$$
\begin{aligned}
\int_{e_{2}}\left|v^{(2)}\right| d s & \leq \int_{\partial B\left(0, h_{1}\right)}\left|v^{(2)}\right| d s \\
& =h_{1} \int_{\partial B(0,1)}\left|\hat{v}^{(2)}\right| d \hat{s} \\
& \lesssim h_{1}\left(\int_{B(0,1)}\left|\nabla \hat{v}^{(2)}\right|^{2} d \hat{x}\right)^{\frac{1}{2}} .
\end{aligned}
$$

This last estimate follows from the property

$$
\hat{v}^{(2)}(\hat{x})=0 \text { on }\left\{\left(x_{1}, 0\right):-1<x_{1}<0\right\},
$$

and the compact embedding of $H^{1}(B(0,1))$ into $L^{2}(B(0,1))$. Coming back to $B\left(0, h_{1}\right)$, we obtain (27).

Using the estimate (27) into (26) and Cauchy-Schwarz inequality, we have shown that

$$
\left|\int_{e} n_{1} v^{(2)} d s\right| \lesssim h_{1}\left\|\nabla v^{(2)}\right\|_{B\left(0, h_{1}\right)}
$$

Let us now pass to the estimate of $\int_{e} n_{2} v^{(1)}(s) d s$ : Denote by $D$ the truncated sector determined by $e$ and the extended crack:

$$
D=\left\{(r \cos \theta, r \sin \theta): 0<r<h_{1} \quad 0<\theta<\theta_{0}\right\},
$$

when we recall that $x_{1}=\left(h_{1} \cos \theta_{0}, h_{1} \sin \theta_{0}\right)$ (see Fig. 6). As before setting $e_{3}=$ $\left\{\left(h_{1} \cos \theta, h_{1} \sin \theta\right): 0<\theta<\theta_{0}\right\}$, we remark that

$$
\int_{D} \partial_{2} v^{(1)} d x=\int_{\partial D} n_{2} v^{(1)} d s=\int_{e} n_{2} v^{(1)} d s+\int_{e_{3}} n_{2} v^{(1)} d s,
$$


because $v^{(1)}=0$ on $\tilde{\Gamma}_{C}=\left\{\left(x_{1}, 0\right): x_{1}>0\right\}$, the extension of the crack $\Gamma_{C}$ to $x_{1}>0$. Hence

$$
\int_{e} n_{2} v^{(1)} d s=\int_{D} \partial_{2} v^{(1)} d x-\int_{e_{3}} n_{2} v^{(1)} d s .
$$

It suffices to estimate the second term of this right-hand side. Again using a scaling argument, we show that

$$
\int_{e_{3}}\left|v^{(1)}\right| d s \lesssim h_{1}\left\|\nabla v^{(1)}\right\|_{B\left(0, h_{1}\right)}
$$

Indeed by construction $v^{(1)}$ satisfies

$$
v^{(1)}=0 \text { on } \tilde{\Gamma}_{C}
$$

Therefore the same scaling arguments as before lead to (30).

Using the estimate (30) into (29) and Cauchy-Schwarz inequality, we have shown that

$$
\left|\int_{e} n_{2} v^{(1)} d s\right| \lesssim h_{1}\left\|\nabla v^{(1)}\right\|_{B\left(0, h_{1}\right)} .
$$

The estimates (28) and (31) into the identity (25) lead to the estimate (24) because $h_{1} \leq h_{K}$.

Let us go on with the Lamé system and recall that in that case $u_{r}^{1}$ and $u_{r}^{2}$ satisfy

$$
\sigma\left(u_{r}^{1}\right) \cdot n=\sigma\left(u_{r}^{2}\right) \cdot n=0 \text { on } \Gamma_{C} .
$$

Since the extension $\tilde{u}_{r}^{1}$ and $\tilde{u}_{r}^{2}$ belong to $H^{2}\left(\bar{\Omega} ; \mathbb{R}^{2}\right)$, they also satisfy the traction free boundary condition on $\Gamma_{C}$, namely

$$
\sigma\left(\tilde{u}_{r}^{1}\right) \cdot n=\sigma\left(\tilde{u}_{r}^{2}\right) \cdot n=0 \text { on } \Gamma_{C} .
$$

Lemma 4 Assume that $x_{1} \in \Omega_{1}$ is a node belonging to a triangle $K$ containing the crack tip. Then the estimate (24) holds.

Proof: The proof starts as before with the identity (25).

We notice that by $(33)$ and since $n=(0,1)^{\top}$ on $\Gamma_{C}, v=\tilde{u}_{r}^{1}-\tilde{u}_{r}^{2}$ satisfies

$$
\begin{aligned}
\lambda\left(\partial_{1} v_{1}+\partial_{2} v_{2}\right)+\mu\left(\partial_{1} v_{2}+\partial_{2} v_{1}\right) & =0 \text { on } \Gamma_{C}, \\
(\lambda+2 \mu) \partial_{2} v_{2}+\lambda \partial_{1} v_{1} & =0 \text { on } \Gamma_{C},
\end{aligned}
$$

where $v_{1}, v_{2}$ are the two components of $v$, i.e., $v=\left(v_{1}, v_{2}\right)^{\top}$. Note that by construction, we also have $v=0$ on $\tilde{\Gamma}_{C}$ and therefore

$$
\partial_{1} v_{1}=\partial_{1} v_{2}=0 \text { on } \tilde{\Gamma}_{C} .
$$

Since $v^{(1)}$ still satisfies

$$
v^{(1)}=0 \text { on } \tilde{\Gamma}_{C},
$$

the arguments of the previous lemma show that (31) is valid.

For the estimate of the term involving $v^{(1)}$, since $n_{1}=0$ on $\Gamma_{C}$, as before the identity (26) holds. To estimate the second term of the right-hand side of (26), we again use a scaling argument: The change of variable $x=h_{1} \hat{x}$ maps $B\left(0, h_{1}\right)$ to the unit ball and by setting $\hat{w}(\hat{x})=\nabla v(x)$, where

$$
\nabla v=\left(\begin{array}{cc}
\partial_{1} v_{1} & \partial_{2} v_{1} \\
\partial_{1} v_{2} & \partial_{2} v_{2}
\end{array}\right)
$$


we deduce that

$$
\begin{aligned}
\int_{e_{2}}\left|v^{(2)}\right| d s & \leq \int_{\partial B\left(0, h_{1}\right)}\left|v^{(2)}\right| d s \\
& \leq \int_{\partial B\left(0, h_{1}\right)}|\nabla v| d s \\
& \leq h_{1} \int_{\partial B(0,1)}|\hat{w}| d s .
\end{aligned}
$$

Now we notice that the conditions (34), (35) and (36) satisfied by $v$ lead to

$$
\begin{array}{r}
\lambda\left(\hat{w}_{11}+\hat{w}_{22}\right)+\mu\left(\hat{w}_{21}+\hat{w}_{12}\right)=0 \text { on }\left\{\left(x_{1}, 0\right):-1<x_{1}<0\right\}, \\
(\lambda+2 \mu) \hat{w}_{22}+\lambda \hat{w}_{11}=0 \text { on }\left\{\left(x_{1}, 0\right):-1<x_{1}<0\right\}, \\
\hat{w}_{11}=\hat{w}_{12}=0 \text { on }\left\{\left(x_{1}, 0\right): 0<x_{1}<1\right\} .
\end{array}
$$

Hence the compact embedding of $H^{1}(B(0,1))$ into $L^{2}(B(0,1))$ and a contradiction argument lead to

$$
\int_{\partial B(0,1)}|\hat{w}| d s \lesssim\|w\|_{1, B(0,1)} \lesssim|w|_{1, B(0,1)} .
$$

This last estimate holds since otherwise we would find a vector field $v \in H^{1}(B(0,1))^{2 \times 2}$ satisfying (37) to (39) such that

$$
|w|_{1, B(0,1)}=0 \text { and }\|w\|_{0, B(0,1)}=1 .
$$

Such a matrix field does not exist because $w$ would be a constant matrix and by (37) to (39), it would be zero.

This estimate leads to (27) and we conclude as in the previous Lemma.

These lemmas allow to treat the non-optimal cases from [12] as follows:

Corollary 1 Let $K$ be a triangle partially enriched and let $K^{*}=K \backslash \Gamma_{C}$. Then

$$
\left\|u-\Pi^{h} u\right\|_{1, K^{*}} \lesssim h_{K}\left(\left|\tilde{u}_{r}^{1}\right|_{2, B\left(0,2 h_{K}\right)}+\left|\tilde{u}_{r}^{2}\right|_{2, B\left(0,2 h_{K}\right)}\right)
$$

Proof: It is sufficient to estimate $\left\|u_{r}-\Pi^{h} u_{r}\right\|_{1, K^{*}}$ since the singular part of $u-\Pi^{h} u$ vanishes. We treat the situation of Fig. 5 (b). Other situations can be treated similarly. We have

$$
\Pi^{h} u_{r}=u_{r}^{1}\left(x_{1}\right) \varphi_{1}+u_{r}^{2}\left(x_{2}\right) \varphi_{2}+\tilde{u}_{r}^{2}\left(x_{3}\right) \varphi_{3} \text { on } K_{2}=K \cap \Omega_{2},
$$

or equivalently

$$
\begin{aligned}
\Pi^{h} u_{r} & =\tilde{u}_{r}^{2}\left(x_{1}\right) \varphi_{1}+u_{r}^{2}\left(x_{2}\right) \varphi_{2}+\tilde{u}_{r}^{2}\left(x_{3}\right) \varphi_{3}+\left(u_{r}^{1}\left(x_{1}\right)-\tilde{u}_{r}^{2}\left(x_{1}\right)\right) \varphi_{1} \text { on } K_{2} \\
& =\Pi^{h} \tilde{u}_{r}^{2}+\left(u_{r}^{1}\left(x_{1}\right)-\tilde{u}_{r}^{2}\left(x_{1}\right)\right) \varphi_{1} \text { on } K_{2} .
\end{aligned}
$$

By the triangular inequality, we may write

$$
\begin{aligned}
\left\|u_{r}-\Pi^{h} u_{r}\right\|_{1, K_{2}} & \leq\left\|u_{r}^{2}-\Pi^{h} \tilde{u}_{r}^{2}\right\|_{1, K_{2}}+\left|u_{r}^{1}\left(x_{1}\right)-\tilde{u}_{r}^{2}\left(x_{1}\right)\right|\left\|\varphi_{1}\right\|_{1, K_{2}} \\
& \lesssim\left\|\tilde{u}_{r}^{2}-\Pi^{h} \tilde{u}_{r}^{2}\right\|_{1, K}+\left|u_{r}^{1}\left(x_{1}\right)-\tilde{u}_{r}^{2}\left(x_{1}\right)\right| .
\end{aligned}
$$

By a standard interpolation error estimate and Lemma 3 (or 4), we conclude that

$$
\left\|u_{r}-\Pi^{h} u_{r}\right\|_{1, K_{2}} \lesssim h_{K}\left(\left|\tilde{u}_{r}^{2}\right|_{2, K}+\left|\tilde{u}_{r}^{1}-\tilde{u}_{r}^{2}\right|_{2, B\left(0, h_{K}\right)}\right) .
$$


For the part on $K_{1}=K \cap \Omega_{1}$, we remark that

$$
\begin{aligned}
\Pi^{h} u_{r} & =u_{r}^{1}\left(x_{1}\right) \varphi_{1}+u_{r}^{2}\left(x_{2}\right) \varphi_{2}+u_{r}^{1}\left(x_{3}\right) \varphi_{3} \text { on } K_{1} \\
& =\Pi^{h} \tilde{u}_{r}^{1}+\left(\tilde{u}_{r}^{1}\left(x_{1}\right)-u_{r}^{2}\left(x_{1}\right)\right) \varphi_{2} \text { on } K_{2} .
\end{aligned}
$$

And we conclude as before because $\tilde{u}_{r}^{1}-u_{r}^{2}$ satisfies the same conditions than $u_{r}^{1}-\tilde{u}_{r}^{2}$ on $\Gamma_{C}$ and $\tilde{\Gamma}_{C}$.

Corollary 2 Let $K$ be the triangle containing the crack tip. Then

$$
\left\|u-\Pi^{h} u\right\|_{1, K^{*}} \lesssim h_{K}\left(\left|\tilde{u}_{r}^{1}\right|_{2, B\left(0, h_{K}\right)}+\left|\tilde{u}_{r}^{2}\right|_{2, B\left(0, h_{K}\right)}\right) .
$$

Proof: In this case we have

$$
\Pi^{h} u_{r}=u_{r}^{1}\left(x_{1}\right) \varphi_{1}+u_{r}^{2}\left(x_{2}\right) \varphi_{2}+u_{r}^{2}\left(x_{3}\right) \varphi_{3} \text { on } K .
$$

Without loss of generality, we may assume that $K$ has one vertex $x_{1}$ in $\Omega_{1}$ and the two other ones $x_{2}, x_{3}$ in $\Omega_{2}$. In this case on $K_{1}=K \cap \Omega_{1}$, we have

$$
\begin{aligned}
\left\|u_{r}-\Pi^{h} u_{r}\right\|_{1, K_{1}} & \leq\left\|u_{r}^{1}-\Pi^{h} \tilde{u}_{r}^{1}\right\|_{1, K_{1}}+\left|u_{r}^{1}\left(x_{2}\right)-\tilde{u}_{r}^{1}\left(x_{2}\right)\right|\left\|\varphi_{2}\right\|_{1, K_{1}}+\left|u_{r}^{1}\left(x_{3}\right)-\tilde{u}_{r}^{1}\left(x_{3}\right)\right|\left\|\varphi_{3}\right\|_{1, K_{1}} \\
& \lesssim\left|\tilde{u}_{r}^{1}-\Pi^{h} \tilde{u}_{r}^{1}\right|_{1, K}+\left\|u_{r}^{1}\left(x_{2}\right)-\tilde{u}_{r}^{1}\left(x_{2}\right)\right\|+\left|u_{r}^{1}\left(x_{3}\right)-\tilde{u}_{r}^{1}\left(x_{3}\right)\right| .
\end{aligned}
$$

We then conclude as in the previous Corollary. The estimate on $K_{2}=K \cap \Omega_{2}$ is treated similarly.

As in [12], these two Corollaries and Lemma 2 lead to the global error estimate of Theorem 1. This analysis is corroborated by the numerical tests also presented in [12]. We reproduce on Fig. 8 the convergence curves obtained in this paper for the approximation (13).

These numerical tests were done on a non-cracked domain defined by $\bar{\Omega}=[-0.5 ; 0.5] \times$ $[-0.5 ; 0.5]$ and the crack was the line segment $\Gamma_{C}=[-0.5 ; 0] \times\{0\}$. The cut-off function $\chi \in C^{2}(\bar{\Omega})$ was defined such that

$$
\left\{\begin{array}{l}
\chi(r)=1 \text { if } r<r_{0}=0.01 \\
\chi(r)=0 \text { if } r>r_{1}=0.49
\end{array}\right.
$$

and $\chi$ was identical to a fifth degree polynomial for $r_{0} \leq r \leq r_{1}$. 


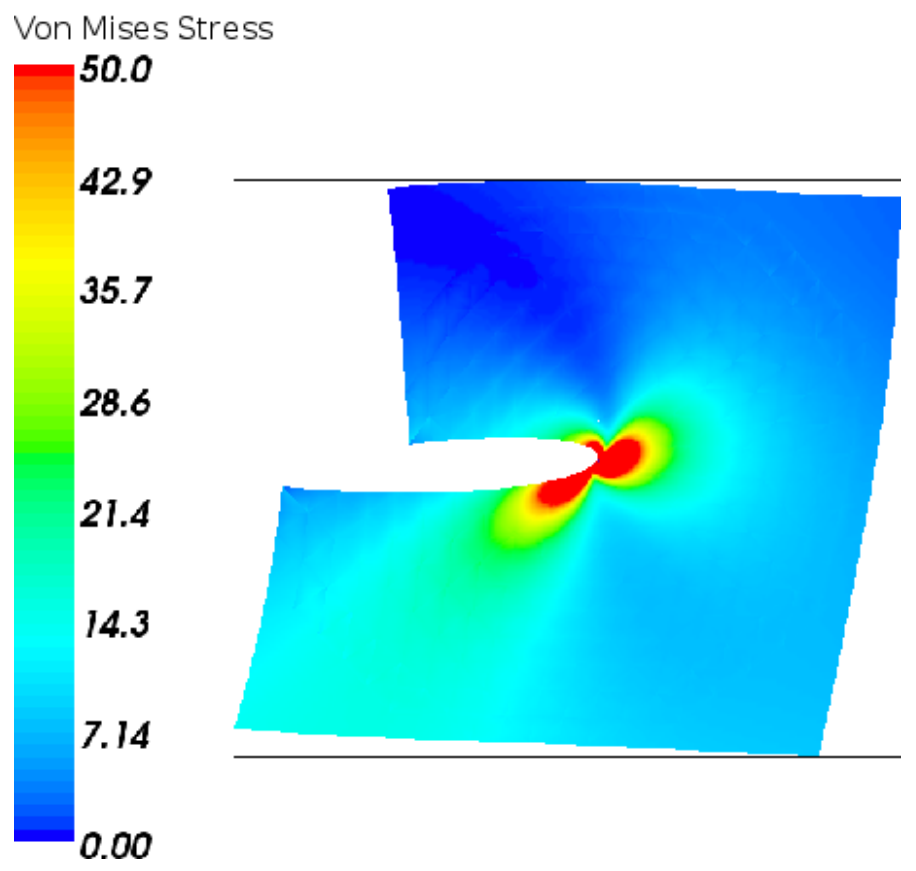

Figure 7: The reference solution, a mixed mode (from [12]).

The exact solution was a combination of a regular solution to the elasticity problem, the mode I and the mode II analytical solutions and a higher order mode (for the deformed configuration, see Fig. 7 with the Von Mises stress). Fig. 8 shows a comparisons of the convergence curves of the non-enriched classical method, the standard Xfem and the cut-off strategy. The optimal rate is obtained for both the cut-off enrichment and the standard Xfem with a fixed enrichment area.

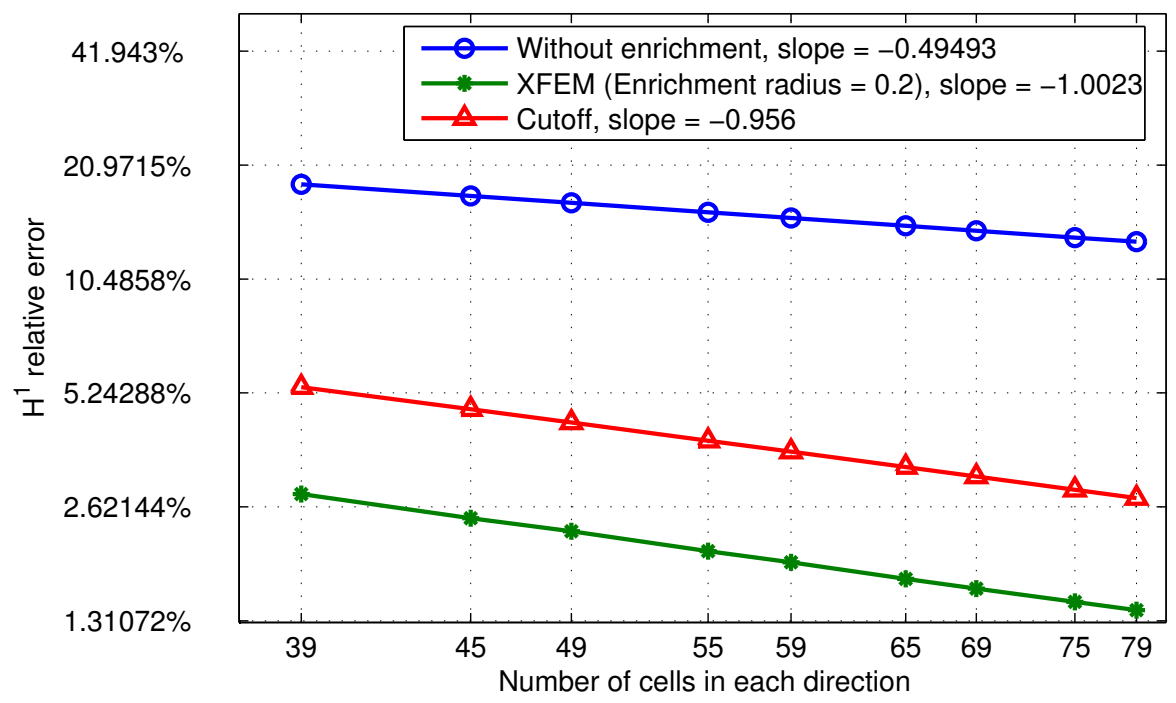

Figure 8: $H^{1}$ error with respect to the number of cells in each direction (ns) for a mixed mode and different enrichment strategies of a P1 elements (from [12]).

Fig. 9 and 10 present some new numerical tests on the comparison between strategies 
(12) and (13) (i.e. between a scalar and a vectorial enrichment) for the same experimental situation. The discrete space corresponding to the scalar enrichment (13) strictly includes the one for the vectorial enrichment (12). However, the gain in $H^{1}(\Omega)$ norm for the error is rather small (Fig. 9). Consequently, the vectorial enrichment appears to be a better choice since the number of additional degrees of freedom is lower and the condition number of the linear system obtained is better (Fig. 10).

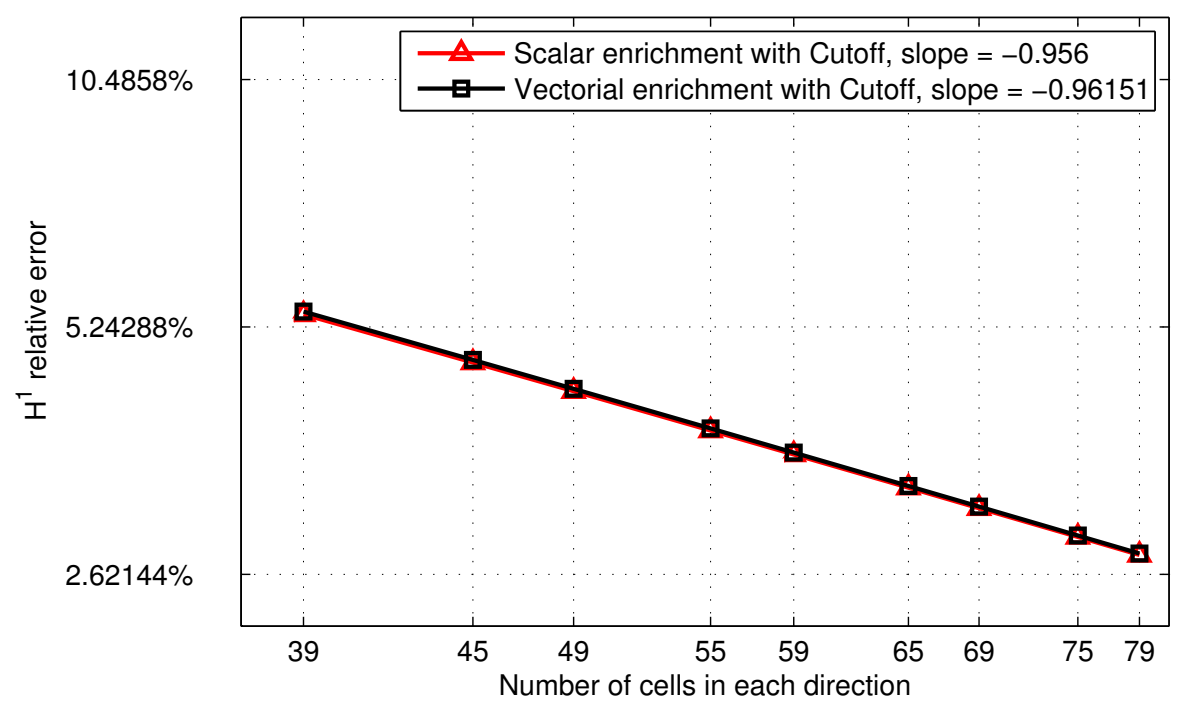

Figure 9: $H^{1}$ error with respect to the number of cells in each direction (ns). Comparison of strategies (12) and (13).

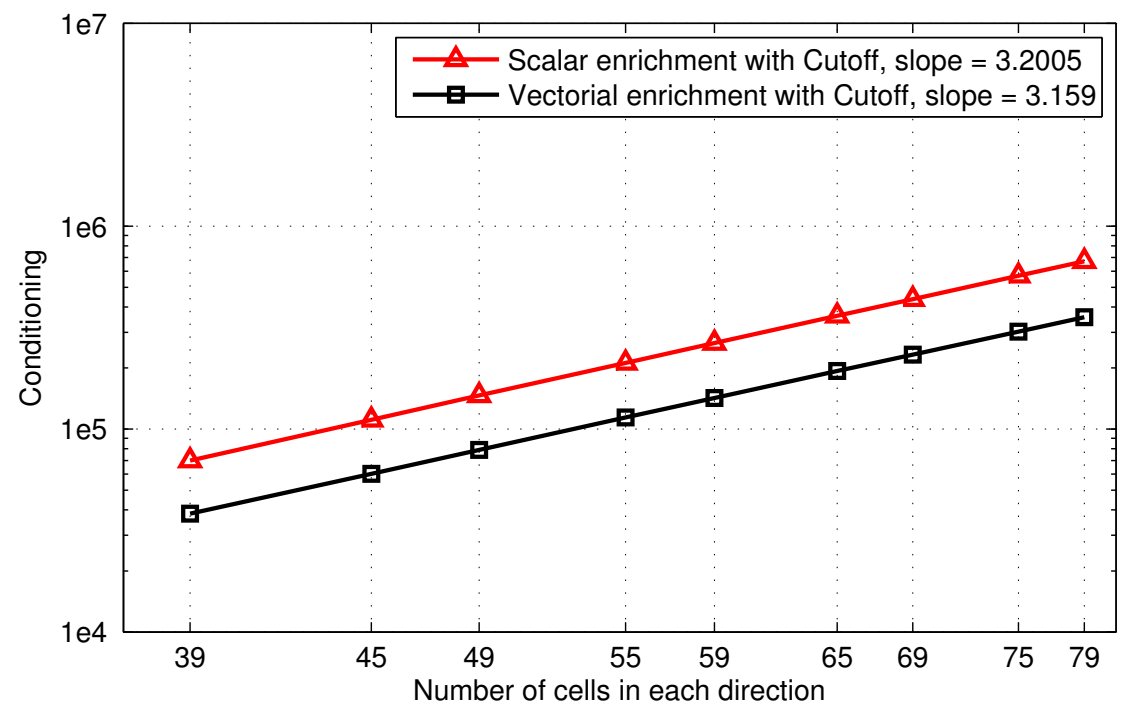

Figure 10: Condition number of the linear system. Comparison of strategies (12) and (13). 


\section{Error estimate on the stress intensity factor}

In this section, we show an error estimate between the exact stress intensity factors and the approximated ones. Let us start with the Laplace equation. Recall that we write

$$
u=u_{r}+K_{L} \chi u_{L},
$$

and that our Galerkin solution $u_{h} \in V_{h}$ solution to (12) admits the splitting

$$
u_{h}=u_{r h}+K_{L, h} \chi u_{L},
$$

where $u_{r h} \in S^{h}$, the space $S^{h}$ being defined by

$$
S^{h}=\left\{v^{h}=\sum_{i \in I} a_{i} \varphi_{i}+\sum_{i \in I_{H}} b_{i} H \varphi_{i} ; a_{i}, b_{i} \in \mathbb{R}\right\},
$$

so that our approximation space $V_{h}$ is spanned by $S^{h}$ plus the singular function $\chi u_{L}$.

Adapting the arguments from Theorem 9.1 of [9] we have the next error estimate:

Theorem 2 Assume that the triangulation is quasi-uniform in the sense that

$$
h \lesssim h_{K} \quad \forall K \in \mathscr{T}_{h} .
$$

Then we have

$$
\left|K_{L}-K_{L, h}\right| \lesssim h^{\frac{1}{2}}
$$

Proof: As in Theorem 9.1 of [9], we have

$$
K_{L}-K_{L, h}=-\frac{a\left(\left(I-G_{h}\right) u_{r},\left(I-G_{h}\right)\left(\chi u_{L}\right)\right)}{a\left(\left(I-G_{h}\right)\left(\chi u_{L}\right),\left(I-G_{h}\right)\left(\chi u_{L}\right)\right)},
$$

where $G_{h} u$ is the Galerkin approximation of $u$ on $S^{h}$, namely $G_{h} u \in S^{h}$ is the unique solution of

$$
a\left(G_{h} u, v_{h}\right)=a\left(u, v_{h}\right) \quad \forall v_{h} \in S^{h} .
$$

By Cauchy-Schwarz's inequality, we deduce that

$$
\left|K_{L}-K_{L, h}\right| \leq \frac{\left\|\left(I-G_{h}\right) u_{r}\right\|_{1, \Omega}}{\left\|\left(I-G_{h}\right)\left(\chi u_{L}\right)\right\|_{1, \Omega}},
$$

Since $u_{r}$ belongs to $H^{2}(\Omega)$ by Theorem 1 , we have

$$
\left\|\left(I-G_{h}\right) u_{r}\right\|_{1, \Omega} \lesssim h\left|u_{r}\right|_{2, \Omega},
$$

and it remains to estimate from below the denominator of (44). For that purpose, we need to adapt the arguments from Lemma 7.1 of [9] because here the triangulation is not aligned with the crack. The main point is to find a small truncated cone $C_{\rho}$ included into the triangle $K$ containing the crack tip with $\rho$ equivalent to $h$. Let us denote by $x_{i}, i=1,2,3$ the three nodes of $K$. First we remark that by a scaling argument we have

$$
\max _{i=1,2,3}\left|x_{i}\right| \geq \frac{\rho_{K}}{\sqrt{2}} \max _{i=1,2,3}\left|\hat{x}_{i}-\hat{O}\right|
$$

where $\left|x_{i}\right|$ is the Euclidean norm of $x_{i}, \hat{O}$ is the pull back of the crack tip $O$ by the affine transformation $F_{K}$ that sends the standard reference element $\hat{K}$ to $K$. Simple calculations show that

$$
\max _{i=1,2,3}\left|\hat{x}_{i}-\hat{O}\right| \geq \frac{1}{4}
$$


and therefore since the triangulation is regular we have

$$
\max _{i=1,2,3}\left|x_{i}\right| \gtrsim h_{K}
$$

We now fix $j \in\{1,2,3\}$ such that

$$
\left|x_{j}\right|=\max _{i=1,2,3}\left|x_{i}\right| \gtrsim h_{K} .
$$

Let $e_{1}$ and $e_{2}$ be the two edges of $K$ having $x_{j}$ as vertex and denote by $\gamma_{\ell}, \ell=1,2$, the angle between $e_{\ell}$ and the segment joining $x_{j}$ to $O$. Without loss of generality we may assume that $\gamma_{1} \geq \gamma_{2}$, and therefore

$$
\gamma_{1} \geq \frac{\alpha_{0}}{2},
$$

where $\alpha_{0} \in\left(0, \frac{\pi}{3}\right)$ is the minimal angle of all triangles of $\mathscr{T}_{h}$ (equivalent to the regularity of the mesh thanks to Zlamal's result [39]).

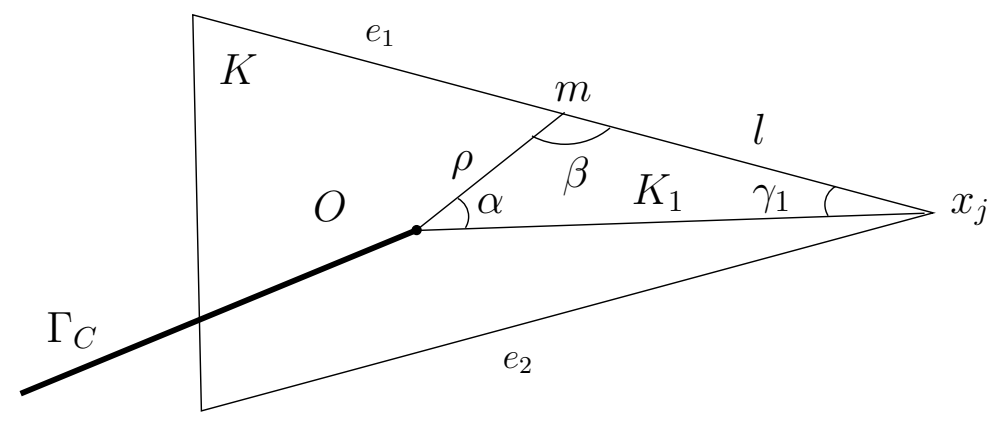

Figure 11: Sub-triangle $K_{1}$.

We now consider the sub-triangle $K_{1}$ of $K$ of vertices $O, x_{j}, m$, where $m$ is the mid-point of the edge $e_{1}$. Denote that $\alpha$ and $\beta$ the angle of $K_{1}$ at $O$ and $m$ respectively (see Fig. 11). Now if $2 l$ is the length of the edge $e_{1}$, by the sinus formula, we notice that

$$
\frac{\sin \alpha}{\sin \beta}=\frac{l}{\left|x_{j}\right|} \sim 1 \text {. }
$$

This property and the fact that

$$
2 \alpha_{0} \leq \alpha+\beta=\pi-\gamma_{1} \leq \pi-\frac{\alpha_{0}}{2},
$$

leads to the existence of a minimal angle $\alpha_{1}>0$ (independent of $h$ ) such that

$$
\alpha>\alpha_{1} .
$$

Denoting by $\rho$ the distance from $O$ to $m$, again by the sinus formula, we have

$$
\rho=\frac{\sin \gamma_{1}}{\sin \alpha} l \sim h_{K},
$$

due to the previous property and the fact that $\alpha \leq \alpha+\beta \leq \pi-\frac{\alpha_{0}}{2}$.

We now denote by $\theta_{O}$ the angle of the half-line containing the segment joining $O$ to $x_{j}$ and consider the truncated cone:

$$
C_{\rho}=\left\{(r \cos \theta, r \sin \theta): 0<r<\rho \quad \theta_{O}<\theta<\theta_{O}+\alpha_{1}\right\} .
$$


By construction, $C_{\rho}$ is included into $K$, and degenerates only in the radial direction. Indeed, by setting $C=\left\{(s \cos \theta, s \sin \theta): 0<s<1 \quad 0<\theta<\alpha_{1}\right\}$ we can introduce the change of variables

$$
F: C \rightarrow C_{\rho}:(s, \omega) \rightarrow\left(\rho s, \theta_{O}+\omega\right) .
$$

Then for every $w_{h} \in V^{h}$ we see that

$$
\left\|\chi u_{L}-w_{h}\right\|_{1, \Omega} \geq\left\|u_{L}-w_{h}\right\|_{1, C_{\rho}} \gtrsim\left|u_{L} \circ F-w_{h} \circ F\right|_{1, C} .
$$

Since $w_{h} \circ F$ belongs to $\mathbb{P}_{1}(C)$, we deduce that

$$
\left\|\chi u_{L}-w_{h}\right\|_{1, \Omega} \gtrsim\left|(I-P)\left(u_{L} \circ F\right)\right|_{1, C},
$$

where $P$ is the projection on $\mathbb{P}_{1}(C)$ with respect to the inner product of $H^{1}(C) / \mathbb{P}_{0}(C)$. Since

$$
u_{L}(r, \theta)=r^{\frac{1}{2}} \sin \frac{\theta}{2}
$$

we have

$$
\begin{aligned}
u_{L} \circ F(s, \omega) & =\rho^{\frac{1}{2}} s^{\frac{1}{2}} \sin \left(\frac{\theta_{O}+\omega}{2}\right) \\
& =\rho^{\frac{1}{2}}\left(\sin \frac{\theta_{O}}{2} S_{D}(s, \omega)+\cos \frac{\theta_{O}}{2} S_{N}(s, \omega)\right),
\end{aligned}
$$

where we have set

$$
S_{N}(s, \omega)=s^{\frac{1}{2}} \sin \frac{\omega}{2} \text { and } S_{D}(s, \omega)=s^{\frac{1}{2}} \cos \frac{\omega}{2} .
$$

With these notations, we have

$$
(I-P)\left(u_{L} \circ F\right)=\rho^{\frac{1}{2}}\left(\sin \frac{\theta_{O}}{2}(I-P) S_{D}+\cos \frac{\theta_{O}}{2}(I-P) S_{N}\right),
$$

and therefore

$$
\left\|\chi u_{L}-w_{h}\right\|_{1, \Omega} \gtrsim \rho^{\frac{1}{2}}\left|\sin \frac{\theta_{O}}{2}(I-P) S_{D}+\cos \frac{\theta_{O}}{2}(I-P) S_{N}\right|_{1, C} .
$$

If we can show that

$$
\left|\sin \frac{\theta_{O}}{2}(I-P) S_{D}+\cos \frac{\theta_{O}}{2}(I-P) S_{N}\right|_{1, C} \gtrsim 1
$$

then

$$
\left\|\chi u_{L}-w_{h}\right\|_{1, \Omega} \gtrsim \rho^{\frac{1}{2}} \gtrsim h^{\frac{1}{2}} .
$$

This estimate with (45) in (44) then lead to the conclusion.

It remains to prove (46). For that purpose, we introduce the function $g$ from $\left[-\frac{\pi}{2}, \frac{\pi}{2}\right]$ into $\mathbb{R}$ defined by

$$
g(\gamma)=\left|\sin \gamma(I-P) S_{D}+\cos \gamma(I-P) S_{N}\right|_{1, C}^{2} .
$$

We first notice that $g(\gamma)>0$ for all $\gamma \in\left[-\frac{\pi}{2}, \frac{\pi}{2}\right]$ simply because $\sin \gamma S_{D}+\cos \gamma S_{N}$ is not a polynomial. Moreover, $g$ is clearly continuous. Therefore

$$
\min _{\gamma \in\left[-\frac{\pi}{2}, \frac{\pi}{2}\right]} g(\gamma)=g\left(\gamma_{0}\right)>0,
$$

for some $\gamma_{0} \in\left[-\frac{\pi}{2}, \frac{\pi}{2}\right]$. The main point is that this minimum is now independent of $\theta_{O}$ and therefore the estimate (46) is proved, and the Theorem follows. 
In the same manner for the Lamé system approximated by (12) we recall that

$$
u=u_{r}+K_{I} \chi u_{I}+K_{I I} \chi u_{I I}
$$

and that our Galerkin solution $u_{h} \in V_{h}$ admits the splitting

$$
u_{h}=u_{r h}+K_{I, h} \chi u_{I}+K_{I I, h} \chi u_{I I}
$$

where $u_{r h} \in\left(S^{h}\right)^{2}$.

As before, we can prove the

Theorem 3 Assume that the triangulation is quasi-uniform in the sense that

$$
h \lesssim h_{K} \quad \forall K \in \mathscr{T}_{h} .
$$

Then we have

$$
\left|K_{I}-K_{I, h}\right|+\left|K_{I I}-K_{I I, h}\right| \lesssim h^{\frac{1}{2}} .
$$

Proof: Following [9], we introduce

$$
V_{I}^{h}=\left(S^{h}\right)^{2} \oplus \operatorname{Span}\left\{\chi u_{I I}\right\} \text { and } V_{I I}^{h}=\left(S^{h}\right)^{2} \oplus \operatorname{Span}\left\{\chi u_{I}\right\},
$$

and denote by $G_{I, h} u$ and $G_{I I, h} u$ the Galerkin approximation of $u$ on $V_{I}^{h}$ and $V_{I I}^{h}$ respectively. By Theorem 9.1 of [9] we know that

$$
\begin{aligned}
K_{I}-K_{I, h} & =-\frac{a\left(\left(I-G_{I, h}\right) u_{r},\left(I-G_{I, h}\right)\left(\chi u_{I}\right)\right)}{a\left(\left(I-G_{I, h}\right)\left(\chi u_{I}\right),\left(I-G_{I, h}\right)\left(\chi u_{I}\right)\right)}, \\
K_{I I}-K_{I I, h} & =-\frac{a\left(\left(I-G_{I I, h}\right) u_{r},\left(I-G_{I I, h}\right)\left(\chi u_{I I}\right)\right)}{a\left(\left(I-G_{I I, h}\right)\left(\chi u_{I I}\right),\left(I-G_{I I, h}\right)\left(\chi u_{I I}\right)\right)} .
\end{aligned}
$$

Therefore by Cauchy-Schwarz's and Korn's inequalities, we have

$$
\begin{aligned}
\left|K_{I}-K_{I, h}\right| & \lesssim \frac{\left\|\left(I-G_{I, h}\right) u_{r}\right\|_{1, \Omega}}{\left\|\left(I-G_{I, h}\right)\left(\chi u_{I}\right)\right\|_{1, \Omega}}, \\
\left|K_{I I}-K_{I I, h}\right| & \lesssim \frac{\left\|\left(I-G_{I, h}\right) u_{r}\right\|_{1, \Omega}}{\left\|\left(I-G_{I, h}\right)\left(\chi u_{I I}\right)\right\|_{1, \Omega}} .
\end{aligned}
$$

The remainder of the proof is the same as the one of the previous Theorem. 


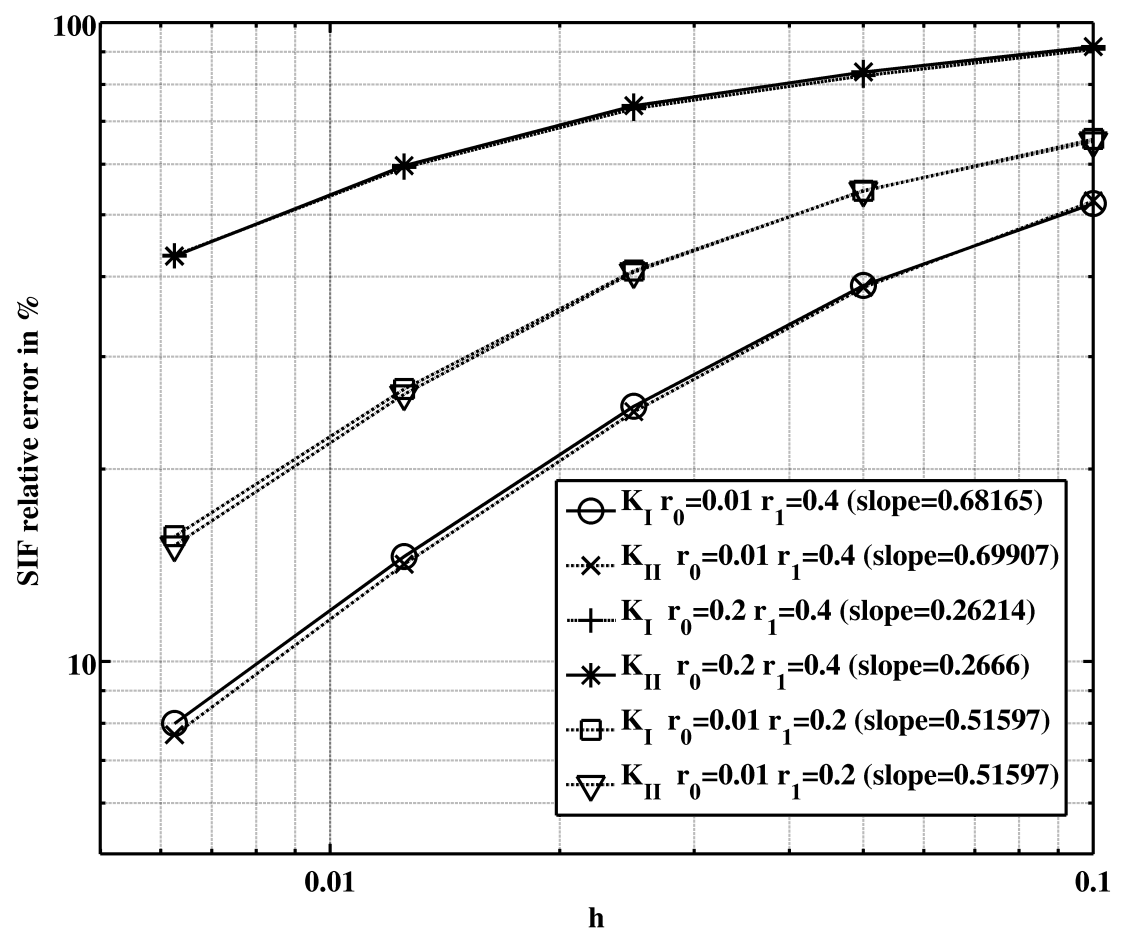

Figure 12: Numerical convergence of stress intensity factors with respect to the mesh parameter $h$.

Let us now present some numerical experiments obtained on the Lamé system with the same reference solution as the one on Fig. 7. The implementation of the discrete problem (12) uses Getfem ++ , the freely available $\mathrm{C}++$ finite element library developed by our team (see [30]). The two stress intensity factors have the same value. The approximation of the stress intensity factor given by $K_{I, h}$ and $K_{I I, h}$ in (12) is presented on Fig. 12. Different values of the radiuses $r_{0}$ and $r_{1}$ corresponding to the definition of the cut-off function (11) are tested in order to show the crucial influence of the shape of the cut-off function. The optimal rate of convergence is reached in the two cases $\left(r_{0}, r_{1}\right)=(0.01,0.4)$ and $\left(r_{0}, r_{1}\right)=(0.01,0.2)$. The sharper is the cut-off function, the worst is the approximation of the stress intensity factors. In the case $\left(r_{0}, r_{1}\right)=(0.2,0.4)$, the optimal rate of convergence is not reached in the range of values of $h$ studied.

This convergence rate is lower than the one obtained by J-integral and interaction integral (see $[18,28]$ for the principle and $[33,34,25]$ for some numerical tests). Such methods require a postprocessing but are superconvergent, for instance in [25] the order of convergence numerically observed for a $P_{1}$ finite element method is close to $O\left(h^{2}\right)$. However, the advantage of the coefficients $K_{I, h}$ and $K_{I I, h}$ of (12) is that they are directly given by the approximation without any postprocessing. Moreover, there is no particular difficulty when the crack tip is near a boundary of the domain.

\section{Optimal error estimate for the standard Xfem}

We give now an a priori error estimate for the standard Xfem with a fixed enrichment area. In the original method proposed in [28] the enrichment with the asymptotic displacement at 
the crack tip is done only on the element containing the crack tip. The rate of convergence of this method is the same than the one without the enrichment (i.e. $O(\sqrt{h})$, see $[31,25])$ since the area of enrichment tends to vanish when the mesh parameter decreases. Of course, this rate of convergence is not difficult to establish. Instead, we prove here an optimal error estimate for the strategy introduced independently in [25] and [3] and called "Xfem with a fixed enrichment area" in the first reference and "Xfem with geometrical enrichment" in the second one and consisting in an enrichment area for the asymptotic displacement whose size is independent of the mesh parameter. The approximation of the Laplace equation with this method reads as

$$
\left\{\begin{array}{l}
\text { Find } u^{h} \in V^{h} \quad \text { such that } a\left(u^{h}, v^{h}\right)=l\left(v^{h}\right) \quad \forall v^{h} \in V^{h}, \\
a\left(u^{h}, v^{h}\right)=\int_{\Omega} \nabla u^{h} \cdot \nabla v^{h} d x, \\
l\left(v^{h}\right)=\int_{\Omega} f v^{h} d x+\int_{\Gamma_{N}} g v^{h} d \Gamma, \\
V^{h}=\left\{v^{h}=\sum_{i \in I} a_{i} \varphi_{i}+\sum_{i \in I_{H}} b_{i} H \varphi_{i}+\sum_{i \in I_{F}} c_{i} \varphi_{i} F_{1} ; a_{i}, b_{i}, c_{i} \in \mathbb{R}\right\},
\end{array}\right.
$$

and the one of the Lamé system is:

$$
\left\{\begin{array}{l}
\text { Find } \quad u^{h} \in V^{h} \quad \text { such that } a\left(u^{h}, v^{h}\right)=l\left(v^{h}\right) \quad \forall v^{h} \in V^{h}, \\
a\left(u^{h}, v^{h}\right)=\int_{\Omega} \sigma\left(u^{h}\right): \varepsilon\left(v^{h}\right) d x, \\
l\left(v^{h}\right)=\int_{\Omega} f \cdot v^{h} d x+\int_{\Gamma_{N}} g \cdot v^{h} d \Gamma, \\
\sigma\left(u^{h}\right)=\lambda \operatorname{tr} \varepsilon\left(u^{h}\right) I+2 \mu \varepsilon\left(u^{h}\right), \\
V^{h}=\left\{v^{h}=\sum_{i \in I} a_{i} \varphi_{i}+\sum_{i \in I_{H}} b_{i} H \varphi_{i}+\sum_{i \in I_{F}} \sum_{j=1}^{4} c_{i, j} \varphi_{i} F_{j} ; a_{i}, b_{i}, c_{i, j} \in \mathbb{R}^{2}\right\} .
\end{array}\right.
$$

where $I_{F}$ is the set of finite element nodes which are inside a disk centered on the crack tip and of a fixed radius $r_{2}$ independent of the mesh parameter. Let us prove now the optimality of this method.

Theorem 4 Assume that the displacement field u, solution to Problem (1) (resp. Problem (2)), satisfies Condition (3). Then, the following estimate holds

$$
\left\|u-u^{h}\right\|_{1, \Omega} \lesssim h\left(\left\|u-u_{s}\right\|_{2, \Omega}+\left\|u_{s}\right\|_{1, \Omega}+\left\|u_{s}\right\|_{2, \Omega \backslash B\left(x^{*}, \frac{r_{2}}{2}\right)}\right),
$$

where $u^{h}$ is the solution to Problem (49) (resp. to Problem (50)).

Proof: Let $\chi$ be a $W^{2, \infty}$ cut-off function satisfying (11) such that $r_{1}<r_{2}$ and $r_{0}>\frac{r_{2}}{2}$. Let

$$
\chi^{h}=I^{h} \chi
$$

be the interpolate of $\chi$ on the $P_{1}$ finite element method. Using the notation of Section 4 , the following interpolation operator

$$
\Pi_{S}^{h} u=I^{h} u_{r}+\chi^{h} u_{s}
$$


clearly satisfies $\Pi_{S}^{h} u \in V^{h}$ for $V^{h}$ defined by (49) (resp. by (50)) at least for $h$ sufficiently small since $r_{1}<r_{2}$. Then,

$$
\begin{aligned}
\left\|\Pi^{h} u-\Pi_{S}^{h} u\right\|_{1, \Omega} & =\left\|\left(\chi-\chi^{h}\right) u_{s}\right\|_{1, \Omega} \\
& \leq\left\|\left(\chi-\chi^{h}\right)\right\|_{W^{1, \infty}}\left\|u_{s}\right\|_{1, \Omega} \\
& \lesssim h\|\chi\|_{W^{2, \infty}}\left\|u_{s}\right\|_{1, \Omega},
\end{aligned}
$$

using a classical error estimate on the interpolation of $\chi$ with a $P_{1}$ finite element method (see for instance [20]). Thus, using the estimates established in section 4, one has

$$
\begin{aligned}
\left\|u-\Pi_{S}^{h} u\right\|_{1, \Omega} & \leq\left\|u-\Pi^{h} u\right\|_{1, \Omega}+\left\|\Pi^{h} u-\Pi_{S}^{h} u\right\|_{1, \Omega} \\
& \lesssim h\left\|u-\chi u_{s}\right\|_{2, \Omega}+h\left\|u_{s}\right\|_{1, \Omega} \\
& \lesssim h\left(\left\|u-u_{s}\right\|_{2, \Omega}+\|1-\chi\|_{W^{2, \infty}}\left\|u_{s}\right\|_{1, \Omega}+\left\|u_{s}\right\|_{2, \Omega \backslash B\left(x^{*}, \frac{r_{2}}{2}\right)}\right)
\end{aligned}
$$

Which ends the proof thanks to Céa's lemma.

Note that this optimal convergence was observed in the numerical results presented on Fig. 8. An interpretation of the proof of Theorem 4 is that the standard Xfem is probably more optimal than the Xfem with a cut-off function because the cut-off function used in the proof is arbitrary. As a consequence, the error bound of the standard Xfem is less than the infimum taken on all the $W^{2, \infty}$ cut-off functions satisfying (11). This is also corroborated with the result on Fig. 8. Of course, the standard Xfem is more expensive than the Xfem with a cut-off function since the number of enrichment degrees of freedom can be greatly higher.

\section{Concluding remarks}

In this paper we have obtained new advances in the analysis of Xfem methods. First, in contrast with [12] we provide optimal a priori error estimates. We also provide an a priori error estimate on the standard Xfem with fixed enrichment area which shows the optimality of this method. As far as we know, this is the first result of this kind for this method. An error estimate on the estimation of the stress intensity factors computed by the variant which uses a cut-off function is also established. We prove that the convergence order is $O\left(h^{1 / 2}\right)$ which is confirmed by numerical experiments. This order is rather low compared to the one obtained with the J-integral (see [18, 33, 34, 28, 25]). However, it permits to have a first estimate without post-treatment of the solution. The numerical experiments show that the quality of the approximated stress intensity factors are very sensitive to the shape of the cut-off function. This suggest to investigate in the future the variant with a pointwise matching [25] or an integral matching [15, 11] which avoid the use of a cut-off function. Another interesting perspective is the generalization to 3D cracks where the computation of the stress intensity factors is more complex. It should be interesting to see if a variant with an integral matching or a cut-off function could be successfully adapted.

\section{References}

[1] R. A. Adams. Sobolev Spaces. Academic Press, 1975.

[2] M. Arfaoui, K. Mansouri, A. Rezgui. An Asymptotic finite plane deformation analysis of the elastostatic fields at a notch vertex of an incompressible hyperelastic material. C.R. Mécanique, 336(9):737-743, 2008. 
[3] E. Béchet, H. Minnebo, N. Moës and B. Burgardt. Improved implementation and robustness study of the X-FEM for stress analysis around cracks. Int. J. Numer. Meth. Engng., 64:1033-1056, 2005.

[4] E. Béchet, N. Moës and B. Wohlmuth. A stable Lagrange multiplier space for stiff interface conditions within the extended finite element method. To appear.

[5] H. Blum, M. Dobrowolski. On finite element methods for elliptic equations on domains with corners. Computing 28(1) (1982) 53-63.

[6] S. Bordas, J.G.C. Conley, B. Moran, J. Gray, and E. Nichols. A simulation-based design paradigm for complex cast components. Engineering with Computers, 23 Issue 1:25-37, 2007.

[7] S. Bordas and B. Moran. Enriched Finite Elements and Level Sets for Damage Tolerance Assessment of Complex Structures. Engng. Fract. Mech., 73:1176-1201, 2006.

[8] S. Bordas, V.P. Nguyen, C. Dunant, H. Nguyen-Dang, and A. Guidoum. An extended finite element library. Int. J. Numer. Meth. Engng., 71(6):703-732, 2007.

[9] M. Bourlard, M. Dauge and S. Nicaise. Error estimates on the coefficients obtained by the singular function method. Numer. Funct. Anal. and Optimiz., 10(11 \& 12):1077$1113,1989$.

[10] M. Bourlard, M. Dauge, M.-S. Lubuma, S. Nicaise. Coefficients of the singularities for elliptic boundary value problems on domains with conical points. III. Finite element methods on polygonal domains. SIAM J. Numer. Anal. 29(1) (1992) 136-155.

[11] E. Chahine. Étude mathématique et numérique de méthodes d'Íḿents finis étendues pour le calcul en domaines fissurés. PhD thesis. INSA Toulouse, France. 2008.

[12] E. Chahine, P. Laborde and Y. Renard. Crack-tip enrichment in the Xfem method using a cut-off function. Int. J. Numer. Meth. Engng., 75(6):629-646, 2008.

[13] E. Chahine, P. Laborde and Y. Renard. Spider-xfem, an extended finite element variant for partially unknown crack-tip displacement. European Journal of Computational Mechanics, 15(5-7):625-636, 2008.

[14] E. Chahine, P. Laborde and Y. Renard. A reduced basis enrichment for the extended finite element method. Math. Model. Nat. Phenom., to appear.

[15] E. Chahine, P. Laborde and Y. Renard. A non-conformal eXtended Finite Element approach: Integral matching XFEM. Submitted.

[16] P. G. Ciarlet. The Finite Element Method For Elliptic Problems. North Holland Publishing Company, 1979.

[17] P. Ciarlet, Jr. and J. He. The singular complement method for 2d scalar problems. $C$. R. Math. Acad. Sci. Paris, 336(4):353-358, 2003.

[18] Ph. Destuynder, M. Djaoua. Sur une interprétation mathématique de l'intégrale de Rice en théorie de la rupture fragile. Math. Meth. in the Appl. Sci., 3:70-87, 1981.

[19] M. Dobrowolski. Numerical approximation of elliptic interface and corner problems. Habilitationsschrift, Bonn 1981. 
[20] A. Ern, J.L. Guermond. Eléments finis: théorie, applications, mise en œuvre. Springer, 2001.

[21] P. Grisvard. Problèmes aux limites dans les polygones - Mode d'emploi. EDF Bull. Directions Etudes Rech. Sér. C. Math. Inform. 1, MR 87g:35073, 21-59, 1986.

[22] P. Grisvard. Singularities in boundary value problems. Masson, 1992.

[23] A. Hansbo, P. Hansbo. A Finite Element Method for the Simulation of Strong and Weak Discontinuities in Solid Mechanics. Comput. Methods Appl. Mech. Engrg., 193:3523-3540, 2004.

[24] J. Haslinger, Y. Renard. A new fictitious domain approach inspired by the extended finite element method. Siam J. on Numer. Anal., to appear.

[25] P. Laborde, Y. Renard, J. Pommier, M. Salaun. High Order Extended Finite Element Method For Cracked Domains. Int. J. Numer. Meth. Engng. 64:354-381, 2005.

[26] J.M. Melenk and I. Babuška. The partition of unity finite element method: Basic theory and applications. Comput. Meths. Appl. Mech. Engrg., 139:289-314, 1996.

[27] N. Moës, T. Belytschko. X-FEM: Nouvelles Frontières Pour les Eléments Finis. Revue européenne des éléments finis (Calcul des structures GIENS'01), 11:305-318, 01/2002.

[28] N. Moës, J. Dolbow, and T. Belytschko. A finite element method for crack growth without remeshing. Int. J. Numer. Meth. Engng., 46:131-150, 1999.

[29] M.-A. Moussaoui. Sur l'approximation des solutions du problème de Dirichlet dans un ouvert avec coins. In Singularities and constructive methods for their treatment (Oberwolfach, 1983), pages 199-206. Springer, Berlin.

[30] Y. Renard, J. Pommier. An open source generic C++ library for finite element methods. http://home.gna.org/getfem/

[31] F.L. Stazi, E. Budyn, J. Chessa, and T. Belytschko. An extended finite element method with higher-order elements for curved cracks. Computational Mechanics, 31:38-48, 2003.

[32] G. Strang, G. Fix. An Analysis of the Finite Element Method. Prentice-Hall, Englewood Cliffs, 1973.

[33] B. A. Szabó, Z. Yosibash. Numerical analysis of singularities in two dimensions. II. Computation of generalized flux/stress intensity factors. Internat. J. Numer. Methods Engrg. 39(3) (1996) 409-434.

[34] B. A. Szabó, Z. Yosibash. Superconvergent extraction of flux intensity factors and first derivatives from finite element solutions. Comput. Methods Appl. Mech. Engrg. 129(4) (1996) 349-370.

[35] G. Ventura. On the elimination of quadrature subcells for discontinuous functions in the eXtended Finite-Element Method. Int. J. Numer. Meth. Engng., 66:761-795, 2006.

[36] E. Wyart, D. Coulon, P. Martiny, T. Pardoen, Jean-Francois Remacle and Frédéric Lani. A substructured FE/XFE method for stress-intensity factors computation in an industrial structure. Revue Européenne de Mécanique Numérique, vol. 16/2:199-212, 2007. 
[37] E. Wyart, D. Coulon, M. Duflot, T. Pardoen, JF Remacle and F. Lani, A substructured FEshell/XFE-3D method for crack analysis in thin walled structures. Int. J. Numer. Meth. Engng., Vol. 72:757-779, 2007.

[38] Q.Z. Xiao, B.L. Karihaloo. Improving the accuracy of Xfem crack tip fields using higher order quadrature and statically admissible stress recovery. Int. J. Numer. Meth. Engng., 66:1378-1410, 2006.

[39] M. Zlámal. On the finite element method. Numer. Math., 12:394-409, 1968. 